\title{
Editorial
}

\section{Diabetic cardiomyopathy: fact or fiction?}

Nearly every physician is familiar with the concept that patients with diabetes mellitus have a heightened cardiovascular mortality rate. ${ }^{1}$ Cardiovascular complications are now the leading cause of diabetes related morbidity and mortality. ${ }^{2}$ It is generally appreciated that the major cardiac complications of diabetes mellitus include the large conduit arteries, epicardial coronary arteries, and the microvasculature. It is also now widely appreciated that the mortality of acute myocardial infarction is greater in patients with diabetes mellitus, ${ }^{3}$ both at 30 days and one year after infarction. Stress hyperglycaemia with and without diabetes mellitus is associated with an increased risk of in-hospital mortality in patients with acute myocardial infarction. ${ }^{4}$

What is less appreciated by physicians and still considered somewhat controversial by some cardiologists is the concept that diabetes mellitus affects cardiac structure and function independent of blood pressure or coronary artery disease. There are now considerable experimental, pathological, epidemiological, and clinical data to support the existence of "diabetic cardiomyopathy". Since the incidence of diabetes mellitus is growing rapidly and is so strongly associated with the development of heart failure, ${ }^{5}$ it is timely to consider the evidence regarding the concept of "diabetic cardiomyopathy", since its initial description nearly 30 years ago. ${ }^{6}$

\section{Experimental findings}

A host of functional and structural abnormalities of the heart muscle have been observed in animal models of diabetes mellitus. ${ }^{78}$ Alloxan induced carbohydrate intolerance in dogs was extensively studied by Regan and colleagues in the 1970s and 1980s. Haemodynamic, biochemical, and histological studies demonstrated a lower stroke volume despite normal left ventricular end diastolic pressure. Chamber stiffness was increased in diabetic dogs compared to control dogs, presumably related to the deposition of interstitial glycoprotein and collagen. These changes could not be prevented by tolbutamide or insulin. Isolated papillary muscle studies in diabetic rats indicate prolongation of contraction, prolonged relaxation, and a reduced rate of shortening. Even rats with non-insulin dependent streptozotocin induced diabetes demonstrate increased left ventricular mass, prolonged isovolumic relation time, elevated left ventricular end diastolic pressure, and increased chamber stiffness. A wide range of abnormal biochemical changes have been described in the hearts of diabetic rats including alterations in ATPase and myosin isoenzymes, impaired calcium ion $\left(\mathrm{Ca}^{2+}\right)$ transport, changes in receptor function, and alterations in carbohydrate, lipid, and adenine nucleotide metabolism. The combination of hypertension and diabetes mellitus is synergistic in rats, and leads to a high mortality, as it does in humans. Experimental animal data overwhelmingly support the connection between diabetes mellitus and heart muscle dysfunction. ${ }^{8}$

\section{Pathology}

It is well established that patients with diabetes mellitus develop coronary artery disease at a younger age, have more multivessel disease, and tend to have more severe, though not more diffuse, coronary artery disease. ${ }^{9}$ Microangiopathic changes in the small vessels of the heart of dia- betic patients may contribute to diabetic cardiomyopathy. ${ }^{10}$ Less well appreciated is the fact that an increase in left ventricular mass is observed in diabetic patients. This is seemingly unrelated to the extent of coronary artery disease. Many patients with diabetes have associated hypertension (28-68\%), so that some of the left ventricular hypertrophy common in diabetics is likely related to high blood pressure. Nevertheless, diabetic women tend to have much greater left ventricular mass, and increased left ventricular wall thickness and chamber size. ${ }^{11}$ Diabetes appears to be an independent contributor to increased left ventricular mass as measured by echocardiography, especially in women. ${ }^{11}$ Other abnormalities noted in human diabetic hearts includes microvascular constriction, interstitial fibrosis, and oedema. ${ }^{10}$ When hypertension is superimposed on the diabetic state, significant myocardial morphologic damage is intensified, producing a powerful substrate for the development of heart failure.

\section{Epidemiological and clinical observations}

Since Rubler and colleagues ${ }^{6}$ first suggested the existence of "diabetic cardiomyopathy" based on postmortem findings in only four adult patients, there has been considerable additional support for the concept from epidemiologic studies $^{15}$ and the clinical literature. ${ }^{7812}$ Diabetes is now well recognised as a risk factor for the development of heart failure. ${ }^{5}$ Diabetic men have more than twice the frequency of heart failure than non-diabetic cohorts, while diabetic women have a fivefold increased risk of developing heart failure. ${ }^{5}$ This excessive risk of heart failure persists despite correcting for age, hypertension, obesity, hypercholesterolaemia, and coronary artery disease. Diabetes mellitus is also a strong risk factor for sudden death (that is, death within one hour of onset of symptoms). ${ }^{13}$ Older studies have suggested the existence of an early or subclinical form of diabetic cardiomyopathy, but contemporary investigations using more physiologically oriented approaches (that is, load and rate independent indexes) indicate no evidence for cardiomyopathy in young adult patients with diabetes who have normal blood pressure and no ischemic heart disease. ${ }^{14}$ The frequency and pace by which preclinical abnormalities progress to clinically evident myocardial dysfunction in patients with diabetes is not established. Moreover, the role of metabolic control of diabetes mellitus in the prevention or the reversal of myocardial dysfunction is unclear. Recent data suggest that non-insulin dependent (type II) diabetics also suffer from increased left ventricular mass and wall thickness and have reduced left ventricular chamber function. ${ }^{15}$ Whatever the mechanisms, the combination of hypertension and diabetes causes substantially more myocardial damage than either entity alone, and remarkably raises the likelihood of developing heart failure.

In summary, diabetes mellitus is associated with increased cardiovascular morbidity and mortality that is likely independent of epicardial and microvascular coronary artery disease. There are ample experimental, pathological, epidemiological, and clinical data to support the existence of a specific "diabetic cardiomyopathy" condition. All patients with diabetes, in addition to 
metabolic control, need aggressive lowering of blood pressure $\leqslant 135 / 80 \mathrm{~mm} \mathrm{Hg}$, and should be treated with an angiotensin converting enzyme (ACE) inhibitor (unless contraindicated) regardless of the level of left ventricular dysfunction when other cardiovascular risk factors are present. ${ }^{16}$ Perhaps the more widespread use of ACE inhibitors in patients with high cardiovascular risk will reduce the propensity to develop diabetes and "diabetic cardiomyopathy", and thus retard the growth of this emerging epidemic. ${ }^{16}$ The role of angiotensin receptor blocking agents for the treatment of hypertension in diabetics is still emerging, but preliminary results are very encouraging.

George $M$ and Linda H Kaufman Center for Heart Failure,

GARY S FRANCIS

Cleveland Clinic Foundation,

9500 Euclid Avenue,

Cleveland $\mathrm{OH}$ 44195, USA

Francig@ccf.org

1 Garcia MJ, McNamara PM, Kannel WB. Morbidity and mortality in diabetics in the Framingham population: sixteen-year follow-up study.

2 American Diabetes Association, the National Heart, Lung, and Blood Institute; the Juvenile Diabetes Foundation International; the National Institute of Diabetes and Digestive and Kidney Disease, and the American Heart Association. Diabetes mellitus: a major risk factor for cardiovascular disease. Circulation 1999;100:1132-3.
3 Mak KH, Topol EJ. Emerging concepts in the management of acute myocardial infarction in patients with diabetes mellitus. $7 \mathrm{Am} \mathrm{Col} \mathrm{Cardiol}$ 2000;35:563-8.

4 Capes SE, Hunt D, Malmberg K, et al. Stress hyperglycemia and increased risk of death after myocardial infarction in patients with and without diabetes: a systemic overview. Lancet 2000;355:773-8.

5 Kannel WB, Hjortland M, Castelli WP. Role of diabetes in congestive heart failure: the Framingham study. Am 7 Cardiol 1974;34:29-34.

6 Rutler S, Dlugash J, Yuceoglu YZ, et al. New type of cardiomyopathy associated with diabetic glomerularsclerosis. Am f Cardiol 1972;30:595-602.

7 Fein FS, Sonnenblick EH. Diabetic cardiomyopathy. Prog Cardiovasc Dis 1985:XXVII:255-70.

8 Fein FS, Sonnenblick EH. Diabetic cardiomyopathy. Cardiovasc Drugs Ther 1994;8:65-73.

9 VanHoeven KH, Factor SM. Diabetic heart disease: the clinical and pathological spectrum - part I. Clin Cardiol 1989;12:600-4.

10 VanHoeven KH, Factor SM. Diabetic heart disease-part II: the clinical and pathological spectrum. Clin Cardiol 1989;12:667-71.

11 Galderisi M, Anderson KM, Wilson PWF, et al. Echocardiographic evidence for the existence of a distinct diabetic cardiomyopathy (the Framingham heart study). Am $\mathcal{f}$ Cardiol 1991;68:85-9.

12 Zarich SW, Nesto RW. Diabetic cardiomyopathy. Am Heart f 1989;118: 1000-12.

13 Balkau B, Jouven X, Ducimetiere P, et al. Diabetes as a risk factor for sudden death. Lancet 1999;354;1968-70.

14 Borow KM, Jaspan JB, Williams KA, et al. Myocardial mechanics in young adult patients with diabetes mellitus: effects of altered load, inotropic state and dynamic exercise. F Am Coll Cardiol 1990;15:1508-17.

15 Devereux RB, Roman MJ, Paranicas M, et al. Impact of diabetes on cardiac structure and function. The strong heart study. Circulation 2000;101:2271-

16 The Heart Outcomes Prevention Evaluation Study Investigators. Effects of an angiotensin-converting-enzyme inhibitor, ramipril on cardiovascular events in high-risk patients. N Engl f Med 2000;342:145-60.

\section{IMAGES IN CARDIOLOGY}

\section{Initiation of pacemaker endless loop tachycardia by triboelectricity}

In the figure below, the three lead ECG on the left shows the initiation of an endless loop tachycardia in a patient with a unipolar DDD pacemaker when the skin over the device was touched at the arrow (low rate $70 \mathrm{ppm}$, upper rate $150 \mathrm{ppm}$, atrioventricular delay $170 \mathrm{~ms}$, postventricular atrial refractory period $200 \mathrm{~ms}$ ). The atrial channel sensed static electricity and
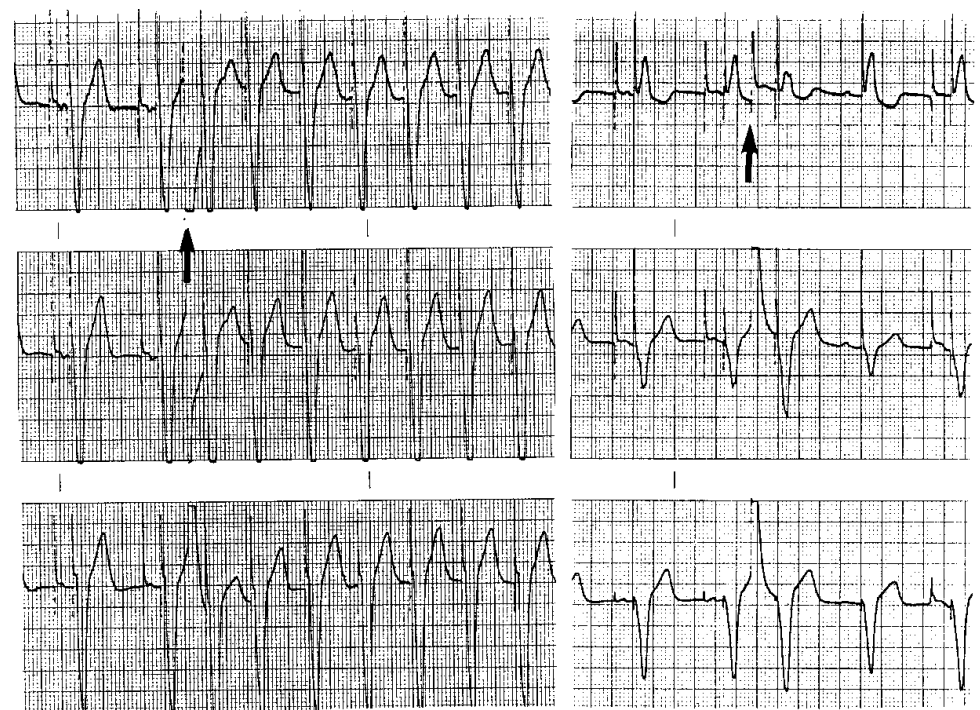

and triggered a ventricular output. The absence of native atrial activity before the paced ventricular beat permitted retrograde ventriculoatrial conduction with initiation of tachycardia.

Static electricity or triboelectric signals may account for mysterious oversensing by unipolar pacemakers. When the relative humidity is quite low, an observer, especially when wearing rubber shoes, may gather considerable static electricity when entering a carpeted room. Touching the patient's skin over a unipolar pacemaker may release sufficient static voltage to be sensed by a unipolar pacemaker. A signal of several millivolts may be sensed by both channels of a $\operatorname{DDD}(\mathrm{R})$ pacemaker and cause inhibition. A smaller signal will be sensed selectively by the atrial channel and trigger a ventricular stimulus as in this case. Triboelectric artefacts may cause puzzling diagnostic problems because they often resemble pacemaker stimuli as shown at the arrow in the three lead ECG on the right recorded from another patient with a unipolar DDD pacemaker. Distortion of the $T$ wave by the applied voltage compounds the situation by producing a pattern that mimicks atrial capture by the triboelectric signal or false atrial stimulus. Two recent reviews on interference with pacemakers made no mention of triboelectric phenomena.

S SERGE BAROLD ssbarold@aol.com 